\title{
GOBERNANZA URBANA, COMPETITIVIDAD Y PROCESOS DE RENOVACIÓN EN LAS CIUDADES MEDIAS ESPAÑOLAS
}

\author{
José Somoza Medina \\ Departamento de Geografía.Universidad de León \\ somoza@unileon.es
}

\section{RESUMEN}

En 1989, el Banco Mundial adoptó el término «gobernanza» para definir nuevas estructuras de cooperación entre el sector público, el sector privado y el denominado tercer sector. Poco tiempo después, la planificación estratégica y la gobernanza urbana se unieron para impulsar grandes proyectos de transformación en determinadas ciudades que aspiraban a mejorar su competitividad, favoreciendo de paso el desarrollo del sector inmobiliario. Veinte años después de los primeros ejemplos, cuando los mismos planteamientos se trasladan a las ciudades medias, el balance esconde muchos claroscuros.

Palabras clave: Gobernanza, competitividad urbana, proyectos de transformación urbana, ciudades medias.

\section{ABSTRACT}

In 1989, the World Bank adopted the term «governance» to define new structures of cooperation among public, private and the «third sector». Shortly thereafter, strategic planning and urban governance came together to promote major transformation projects in selected cities who wanted to improve their competitiveness, encouraging in parallel the real estate development. Twenty years after the first examples, when these approaches are transferred to medium cities, the balance hides many contrasts.

Key words: Governance, urban competitiveness, urban transformation projects, mediumsized cities.

Fecha de recepción: abril 2011

Fecha de aceptación: diciembre 2012. 
En este tiempo de cambios rápidos y transformaciones sociales impulsadas en gran medida por una situación de crisis económica mundial, la gobernanza es claramente un arma de doble filo, como concepto impulsado por los organismos supranacionales y las organizaciones políticas conservadoras. La difusión interesada por estos entes de la retórica de la «gobernanza» en las estructuras de gobierno municipal pretende impulsar un nuevo modelo de gestión pública multifactorial y en red, en el que el capital privado tenga, cada vez, un papel más preponderante.

En las metrópolis de todo el mundo ya es una realidad vigente desde la última década y ahora, con mayor lentitud y sin los grandes proyectos de transformación urbana detrás, empieza a introducirse en el discurso político de las ciudades intermedias. La excesiva reiteración de los términos eficacia, eficiencia, pragmatismo y austeridad en el gasto referidas a las corporaciones municipales, trasladan a la opinión pública la idea de que son los parámetros económicos los indicadores más importantes para juzgar una determinada opción política asentada en el gobierno de la ciudad. Para esta visión de la realidad, que se expande mejor en épocas de carestía, no es posible el desarrollo sin crecimiento ni el aumento de calidad de vida sin un incremento paralelo del nivel de vida. Entramos de esta forma en una espiral en la que lo crucial es negociar con el capital privado como mejorar nuestro producto, la ciudad, para que tenga una posición ventajosa en el mercado internacional de urbes.

En base a estas ideas, puede ser el momento apropiado para revisar los conceptos de gobernanza y competitividad urbana, estudiar sus características y tratar de reorientar su implementación en la agenda ciudadana. Estos son los objetivos de este artículo.

\section{GOBERNANZA. UN CONCEPTO IDEOLOGIZADO Y EN EVOLUCIÓN}

Los autores que han analizado la gobernanza ofrecen diversas interpretaciones sobre el proceso de conceptualización de este vocablo. La palabra empieza a tener difusión a finales de los años 1970 en relación a la política, gestión y administración interna de las empresas privadas (corporate governance) por parte de la escuela de «neoinstitucionalistas» angloamericanos, próxima a su vez a la escuela de economistas de Chicago, con Milton Friedman a la cabeza, que defendía la extensión de los métodos de la economía neoclásica al resto de las ciencias sociales (García, 2009). Tiempo después, el término es utilizado por el «think tank» neoliberal Instituto de Finanzas, que impulsa el llamado Consenso de Washington, a partir del cual el Banco Mundial y el Fondo Monetario Internacional asumen conceder préstamos sólo a los países pobres que establecieran sistemas de gobernanza, es decir, nuevas fórmulas de gobierno caracterizadas por la eficacia y la eficiencia, con medidas como la reducción de los presupuestos asignados a servicios públicos para los sectores más necesitados, recortes en la educación y en la sanidad públicas e incluso su privatización, eliminación de subvenciones y su substitución por préstamos, todo ello buscando que las políticas públicas sean las garantes del buen funcionamiento del mercado (Conejero, 2005). Las estrategias de lucha contra la pobreza que defienden estos organismos internacionales pasan desde entonces por la injerencia en las economías nacionales de estos países con intensos programas de ajuste económico. La mejor gobernación (better governance) a la que se refiere el Banco Mundial en el informe de noviembre de 1989 sobre África subsahariana vincula directamente gobernanza y desarrollo, lo mismo que hace poco tiempo después la OCDE (Rojo, 2009). 
En la década de los años 1990, el Programa de las Naciones Unidas para el Desarrollo (PNUD) dulcificó el término añadiendo un adjetivo al hablar de «buena» gobernanza (good governance), que se genera reclamando una menor intervención estatal en la canalización de la asistencia financiera a los países no desarrollados y una mayor participación de la «sociedad civil». Se reduce de esta manera la excesiva verticalidad en los modelos de cooperación internacional para facilitar la horizontalidad, introduciendo al sector privado y al denominado «tercer sector» en procesos de tomas de decisiones en los que interactúa con el sector público.

En 2001 es la Comisión Europea la que designa a la gobernanza como el modelo ideal de funcionamiento de las instituciones comunitarias, y por extensión, del resto de instituciones públicas, tanto locales, como regionales, estatales o internacionales. En el libro blanco se define la gobernanza europea como las normas, procesos y comportamientos que influyen en el ejercicio de los poderes a nivel europeo, especialmente desde el punto de vista de la apertura, la participación, la responsabilidad, la eficacia y la coherencia. Estos últimos son para la Comisión Europea los cinco principios de la buena gobernanza: apertura en cuanto a la transparencia en las actuaciones de las instituciones para lograr una mayor confianza y comunicación con el público en general, participación de la ciudadanía en todas y cada una de las distintas fases del proceso político, responsabilidad de todas las instituciones comunitarias pero también de los Estados y de todos los agentes que participan en el desarrollo y la aplicación de las políticas europeas, eficacia en la aplicación proporcionada de recursos para provocar los resultados buscados sobre la base de unos objetivos claros, la evaluación de su futuro impacto y la experiencia acumulada, y finalmente coherencia en las políticas desarrolladas y las acciones emprendidas (Comisión Europea, 2001).

En esta línea se sitúa también la definición que introdujo la Real Academia Española en su diccionario en el año 2000; arte o manera de gobernar que se propone como objetivo el logro de un desarrollo económico, social e institucional duradero, promoviendo un equilibrio sano entre el Estado, la sociedad civil y el mercado de la economía.

Estas definiciones caminan intencionadamente hacia una desideologización del concepto, reduciéndolo a una estructura de relaciones entre los diferentes actores a través de la cual se toman las decisiones sobre lo público (Centelles, 2006). Ismael Blanco y Ricard Gomá (2006) apuestan por una caracterización del concepto en base a tres proposiciones: el reconocimiento, la aceptación y la integración de la gestión del conflicto como un elemento intrínseco del proceso político, un sistema de gobierno a través de la participación de actores diversos en el marco de redes plurales, y una nueva posición de los poderes públicos en los procesos de gobierno, con la adopción de nuevas funciones y la utilización de nuevos instrumentos de gestión.

Como afirma Joaquín Farinós, el carácter polisémico de este concepto permitió que fuera adoptado por escuelas de pensamiento muy divergentes, coma la Nueva Gestión Pública de los neoliberales, el nuevo estado social de los neoinstitucionalistas, o incluso el universalismo comunitario de la sociedad civil y de las organizaciones no gubernamentales (Farinós, 2008). Para este geógrafo pueden distinguirse cuatro dimensiones de la gobernanza: la gobernanza multinivel entre diferentes sistemas político-administrativos, la gobernanza como cooperación horizontal entre políticas sectoriales y territorios, la gobernanza económica a través de las redes de actores sociales y empresas, y la participación ciudadana (Farinós, 2006). 
R.A.W. Rhodes define la gobernanza como un modelo emergente de regulación social e identifica siete usos diferenciados del concepto: gobernanza corporativa, nueva gestión pública, buena gobernanza, relaciones internacionales, sistema socio-cibernético de gobierno, nueva política económica con influencias marxistas y gobernanza por redes (Rhodes, 1996). En sus investigaciones más recientes (Bevir e Rhodes, 2010) explica como hoy en día es más preciso hablar de tres olas consecutivas de gobernanza en la administración pública, de la gobernanza en redes a la metagobernanza y de esta a la gobernanza descentralizada. Algunos autores hablan incluso de la gobernanza sin gobierno (Rosenau y Czempiel, 1992; Rhodes 1996), de la legitimidad democrática de la gobernanza (Martínez, 2010) o de asociaciones de palabras en origen tan antitéticas como gobernanza territorial para el desarrollo sostenible (Farinós, 2008).

Jan Kooiman, que en 2005 definía diez modelos diferentes de gobernanza (Kooiman, 2005), en su primera aproximación a este concepto afirmaba que la única forma de gobernar sistemas sociopolíticos dinámicos, diversos y complejos de un modo democrático y efectivo es incorporar el dinamismo, la diversidad y la complejidad de nuestras sociedades (Kooiman, 1993). La globalización, la mundialización de la economía, la nueva sociedad posmoderna, la crisis fiscal y política, en definitiva el territorio convulso en el que se desarrolla el ser humano desde finales del pasado siglo parecen conducir inexorablemente a la necesidad de un nuevo sistema de gobierno, llamado gobernanza, en el que los dueños del capital ya no tienen porque esconderse detrás de los políticos, sino posicionarse directamente a su lado y orientar las políticas públicas. En palabras de Enrique Conejero, la gobernanza se inserta en un escenario en el que prevalece la fragmentación institucional, una red de políticas públicas complejas y unas fronteras difusas entre el sector público, el privado y la sociedad civil (Conejero, 2005).

Esa ambigüedad es denunciada por algunos autores que pretenden restituir la preponderancia que debe asumir el sector público y por ello hablan de una gobernanza reforzada (Romero y Farinós, 2011), en la que el Estado conserve el papel motor y preeminente sobre los otros dos componentes. Un nuevo modelo de gobernanza democrática, definido como un modo de gobernar basado en la horizontalidad y el acuerdo (Pascual y Godás, 2010).

De esta forma, la gobernanza va introduciéndose en las instituciones políticas siguiendo una escala de mayor a menor complejidad, de las relaciones mundiales entre países ricos y países pobres a los organismos supranacionales continentales, luego a los gobiernos estatales, regionales y finalmente a las corporaciones municipales, primero a las de las grandes metrópolis y después a las del resto. Pasamos así de la gobernanza territorial a la gobernanza urbana, visible especialmente en los procesos de transformación experimentadas por las principales ciudades mundiales en estos últimos 25 años.

De hecho, algunos autores (Rodríguez, Moulaert y Swyngedouw, 2001) sostienen que el término «gobernanza urbana» substituyó ya al de «gobierno urbano» para referirse a la creciente complejidad de la intervención pública en la ciudad. Según estos autores, las ciudades se rigen ahora por estructuras relacionales entre los órganos tradicionales de los gobiernos locales y otras instituciones y asociaciones del sector privado, las ONG y la ciudadanía. Esta gobernanza urbana motiva procesos de descentralización y fragmentación de responsabilidades siguiendo un modelo productivista, orientado por criterios de eficiencia en la provisión de servicios y la subordinación de objetivos sociales redistributivos a los imperativos de la 
competitividad y la promoción del crecimiento económico (Jessop, 1997). La buena gobernanza urbana implica establecer lazos de cohesión entre los diferentes sectores, público, privado, voluntariado y ciudadanía, a través del desarrollo de estructuras y procesos que refuercen la colaboración y se caractericen por el liderazgo efectivo y la participación inclusiva (Haus, Heinelt e Stewart, 2005). Para Bernard Jouve, la coalición público-privada en el gobierno de la ciudad sólo es posible si la caracterización política de su base productiva y los canales de mediación entre entidades locales y actores privados son estables en el tiempo (Jouve, 2005).

Tabla 1

LAEVOLUCIÓN DE UN CONCEPTO

\begin{tabular}{|l|l|l|l|}
\hline \multicolumn{1}{|c|}{ Año } & \multicolumn{1}{|c|}{ Término } & \multicolumn{1}{c|}{ Procedencia } & \multicolumn{1}{c|}{ Significado } \\
\hline $\begin{array}{l}\text { Finales } \\
1970\end{array}$ & $\begin{array}{l}\text { Corporate } \\
\text { governance }\end{array}$ & $\begin{array}{l}\text { Neoinstitucionalismo } \\
\text { Económico }\end{array}$ & $\begin{array}{l}\text { Política, gestión y administración } \\
\text { interna de las empresas privadas }\end{array}$ \\
\hline $\begin{array}{l}\text { Finales } \\
1980\end{array}$ & Governance & $\begin{array}{l}\text { Neoliberalismo } \\
\text { Económico }\end{array}$ & $\begin{array}{l}\text { Nuevas fórmulas relacionales de } \\
\text { gobierno con la participación del } \\
\text { sector privado y caracterizadas por } \\
\text { la eficacia y la eficiencia económica. }\end{array}$ \\
\hline 1989 & $\begin{array}{l}\text { Better } \\
\text { governance }\end{array}$ & Banco Mundial & $\begin{array}{l}\text { Estructuras relacionales en las que } \\
\text { se incluye el sector público, el sec- } \\
\text { tor privado y la sociedad civil para } \\
\text { promover el desarrollo económico }\end{array}$ \\
\hline 1997 & $\begin{array}{l}\text { Good } \\
\text { governance }\end{array}$ & PNUD & $\begin{array}{l}\text { Eficacia, transparencia, participa- } \\
\text { ción, } \\
\text { Equidad y rendición de cuentas. }\end{array}$ \\
\hline 2001 & $\begin{array}{l}\text { Good European } \\
\text { Governance }\end{array}$ & Comisión Europea & $\begin{array}{l}\text { Apertura, participación, responsabi- } \\
\text { lidad, eficacia y coherencia. }\end{array}$ \\
\hline
\end{tabular}

La gobernanza, por tanto, es un concepto ideologizado y polisémico de origen neoliberal que pretende normalizar la creación de estructuras relacionales público-privadas para dirigir las políticas, proyectos y gestiones de las instituciones democráticas (García, 2009). Una dinámica preocupante que desde el punto de vista académico parece haber tocado techo (Romero y Farinós, 2011) y que puede resolverse definitivamente en una gobernanza sin gobierno (pensemos en las situaciones recientes de Italia o Grecia) o retomar los valores democráticos y de participación ciudadana que deben cimentar los buenos gobiernos del siglo XXI.

\section{RENOVACIÓN Y COMPETITIVIDAD URBANA. ALGO MÁS QUE UN CAMBIO DE IMAGEN}

Arquitectos y geógrafos han analizado las transformaciones sufridas por las ciudades en todo el mundo desde los ejemplos pioneros de los años 1980. La intervención urbana a gran escala en los espacios vacíos de las ciudades centrales comienza en Estados Unidos en lugares como Baltimore, Cleveland o Pittsburg, y se traslada posteriormente al resto del mundo, con casos estudiados en Glasgow, Londres, París, Berlín, Rotterdam, Stuttgart, Bir- 
mingham, Hong Kong, Sao Paulo, Buenos Aires, Tokio, Newcastle, Lyon, Singapur, Turín, Bogotá, Lille, Milán, Guayaquil, Newcastle, o Santiago de Chile (Davies e Townshend 2002; Pacione, 2004; Pack, 2004; Murayama e Du, 2005; Lois, 2006), y en el caso de España con los ejemplos fundamentalmente de Bilbao, Barcelona, Valencia, Zaragoza, Madrid y Sevilla. En todos estos casos, las intervenciones estuvieron ligadas a estrategias de reconstrucción de la imagen y promoción de la ciudad, la apuesta por reforzar la competitividad urbana y atraer futuras inversiones.

El modelo fue casi siempre el mismo, áreas degradadas y abandonadas por el declive de la industria, la descentralización y reestructuración productiva o los cambios en las grandes infraestructuras de transportes, fueron intervenidas urbanísticamente para crear, en espacios apetecibles para el mercado, un nuevo y exclusivo pedazo urbano que pretende representar a la totalidad de su imagen de ciudad en el siglo XXI (Etulian, 2008). Las operaciones urbanísticas creadoras de «nuevas centralidades» combinan usos productivos, residenciales, comerciales, culturales y de ocio donde se integran un conjunto de elementos recurrentes y diseñados siguiendo un mismo patrón con arquitecturas emblemáticas, proyectos de espectacularización del espacio público, centros de convenciones, infraestructuras culturales y turísticas, parques temáticos, festivales y otros eventos internacionales con una finalidad propagandística y de marketing urbano (Ashworth y Voogd, 1990).

Las grandes intervenciones se publicitaban inicialmente siempre como operaciones autofinanciadas por la repercusión sobre los presupuestos del incremento del precio del suelo y las consecuentes aportaciones de las grandes empresas inmobiliarias, aunque por lo general los proyectos terminaban con importantes sobrecostes a los que se enfrentaban solidariamente los diferentes niveles administrativos implicados (gobiernos locales, regionales y estatales) pero nunca las empresas constructoras, que eran las que hacían el verdadero negocio.

Los grandes proyectos urbanos fueran ya criticados por David Harvey en 1989, pues empleaban inversiones públicas directas e incentivos fiscales y financieros para estimular la inversión privada, mostrando el cambio de orientación en las prioridades de la planificación y las políticas urbanas, caracterizadas ahora por el ascenso de un estilo de gobierno poskeynesiano que incorporaba formas corporativas a través de la adopción de métodos empresariales por parte de las instituciones gubernamentales. De esta forma, las grandes ciudades se gestionaban como si fueran negocios y más que responder a las necesidades de la ciudadanía, el objetivo era ahora ser competitivas en las redes de flujos internacionales (Harvey, 1989).

Es decir, ya en la década de los años 1980, los primeros proyectos de transformación urbana revelaban el mismo camino que la cooperación internacional o las recomendaciones de los organismos supranacionales, establecer fórmulas público-privadas para favorecer al mercado con inversiones públicas en el marco de nuevas fórmulas de gobernanza local.

La planificación estratégica contribuyó a consolidar esta visión de los grandes proyectos urbanos como motores y orientadores del desarrollo urbano en el marco de una relación dialéctica, no secuencial, entre objetivos, estrategias y proyectos. Una planificación que permitía superar los esquemas demasiado rígidos de la ordenación urbanística, e introducir el lenguaje y la ideología empresarial en los gobiernos urbanos. La reorientación estratégica hacia el urbanismo empresarial asumía así la existencia de la competencia entre ciudades y, en consecuencia, la lógica búsqueda de ventajas competitivas tratando de proyectar una imagen de ciudad dinámica e innovadora, estimulante y creativa, capaz de competir con éxito 
por la atracción de nuevas inversiones productivas y consumidores, bien turistas o nuevos residentes (Rodríguez, Moulaert y Swyngedouw, 2001).

Lo paradójico es que la repetición en serie de los mismos instrumentos y artefactos, desde el diseño de la arquitectura y la elección de los arquitectos-estrella hasta los slogans promocionales, reintrodujeron la homogeneidad y la indiferenciación entre lo que pretendían ser proyectos emblemáticos y exclusivos. Más que introducir diferencias acababan creando paisajes semejantes (Harvey, 1989).

Peter Hall, otro reconocido geógrafo urbano, afirmaba en 1988 que los procesos de renovación urbana eran respuestas empresariales a las nuevas oportunidades de desarrollo, que «colonizaban» la agenda urbana con criterios emprendedores (Hall, 1988). Estas operaciones tenían una clara orientación estratégica en la medida en que la reurbanización de suelos degradados se percibía como una oportunidad excepcional para dotar a la ciudad de las condiciones necesarias para acoger nuevos usos y funciones dinamizadoras capaces de relanzar una nueva fase de crecimiento urbano (Hall, 1995).

Las crisis de crecimiento que presentaban las ciudades en los años 1980 y 1990 se trataron de solucionar creando nuevas combinaciones de factores económicos que hicieran más competitivas a las ciudades. Estas teóricamente nuevas combinaciones recibieron como conceptualización diversos y expresivos nombres, «empresarialismo urbano», «corporatismo», «emprendedorismo» o simplemente «gobernanza» (Jessop, 1997; Hall e Hubbard, 1998; Jouve, 2005).

En esta nueva etapa, las ciudades se entienden cada vez más como actores principales del desarrollo económico, como motores dinámicos, y no como meros contenedores de acción social. Si antes el gobierno de la ciudad se definía coma la gestión de los bienes y servicios públicos y la respuesta a las necesidades de los ciudadanos, ahora los gobiernos locales se comportan como promotores urbanos (González, 2007). La política urbana pasó de ocuparse de la provisión de servicios, a la gestión del crecimiento y el control de las externalidades negativas, a intervenir para impulsar el crecimiento económico y apoyar el desarrollo de nuevas funcionalidades urbanas (Hall, 1988). De la misma manera, la práctica urbanística ya no se orienta a minimizar las consecuencias negativas del crecimiento urbano mediante mecanismos de redistribución, ahora se trata de maximizar las oportunidades en un contexto de recursos reducidos y en condiciones de creciente competencia interurbana (Albrechts, 1992).

La nueva agenda urbana adopta un estilo proactivo, dinámico y empresarial, buscando oportunidades donde el sector público y el privado puedan concertar acciones conjuntas, llegando a la excesiva reiteración del término competitividad en contextos donde se pretenden justificar grandes inversiones que carecen de una apropiada planificación y de un análisis pausado de su verdadero impacto futuro (Lois, 2010).

La retórica de la competitividad orienta a las autoridades locales hacia unas políticas de atracción de inversiones desde fuera, en vez de centrarse en valorizar los recursos endógenos (González, 2007). Las nuevas políticas urbanas reflejan la prioridad de la búsqueda del crecimiento y la regeneración urbana subordinando los objetivos sociales a la lógica de la competitividad. Al fin y al cabo, en un escenario dirigido por el mercado los ciudadanos sólo tienen derechos como productores o consumidores (García, 2009). Aunque debería resultar imposible comparar un gobierno local prestatario de servicios sociales con una fábrica de 
coches, o una ciudad con una empresa, pues no sabemos cuál es su negocio, ni es factible que cierre si le va mal la cuenta de resultados (González, 2007; García 2009).

Lo que parece evidente es que la expansión de las ideas neoliberales provocó que las grandes ciudades comenzaran a guiarse por criterios de competitividad (Somoza, 2007). El nuevo objetivo de las corporaciones es reforzar la capacidad local para enfrentar con éxito la competencia global, lo que se convierte en una tarea inacabable. La competitividad se transforma en el principal argumento de las políticas urbanas en la globalización y en el fin último de la gestión local de la economía global (Borja y Castells, 1997).

La globalización pretende ser la escusa de la nueva gobernanza urbana, cuando en realidad las actuaciones concertadas del sector privado y del sector público en los grandes proyectos de transformación urbana son ellos mismos procesos generadores de globalización económica, transnacionalización cultural y competencia interurbana.

En estas operaciones urbanísticas, los gobiernos lideran de manera proactiva una nueva política económica basada en grandes proyectos estelares, promoviendo las inversiones privadas por medio de instrumentos de apoyo directo e indirecto, incentivos fiscales y costosas campañas de marketing urbano. Las estrategias se articulan en base a tres tendencias interdependientes: el desplazamiento de las prioridades de la intervención urbana hacia el crecimiento y la restructuración competitiva; la reorientación del enfoque predominantemente gestor y regulador de la política urbana hacia un enfoque proactivo y empresarial; y los cambios en la instrumentación de la intervención y la aparición de un nuevo modo de gobernanza urbana (Rodríguez, Moulaert e Swyngedouw, 2001).

Como vemos, son muchas las ciudades que introducen en su discurso estrategias de posicionamiento en el escenario internacional, grandes proyectos de concertación con la sociedad civil y los principales actores económicos, o nuevas oportunidades de transformación con la designación de un evento o nombramiento de carácter internacional (olimpiadas, exposiciones universales, ciudad cultural europea, ciudad patrimonio de la humanidad, etc.) que permitan proyectar la «nueva» identidad de esa ciudad al escaparate del mercado mundial.

\section{PRIMEROS BALANCES SOBRE LOS PROCESOS DE TRANSFORMACIÓN Y GOBERNANZA EN ALGUNAS CIUDADES}

Cuando ya han transcurrido más de 20 años desde los primeros proyectos de recuperación urbana, el balance de resultados no es ni mucho menos alentador para aquellas ciudades que dirigieron su estrategia de crecimiento hacia la concentración de inversiones y proyectos de espectacularización en un área determinada. En los tres casos señalados de Estados Unidos: Baltimore, Cleveland y Pittsburgh, las reformas consiguieron crear en los espacios reformados auténticas islas de diseño, lujo y arquitectura de vanguardia. Los procesos de gentrificación se extendieron por estas áreas, disparando los precios de las viviendas, pero la atracción de inversiones, empresas y población no se produjo. Eso sí, para la revista The Economist, y concretamente según su Unidad de Inteligencia Económica, en 2005 Cleveland y Pittsburgh eran las dos primeras ciudades estadounidenses en el ranking de habitabilidad. Por cierto, los rankings y las posiciones dentro de ellos son una de las máximas expresiones de la competitividad urbana. Estos rankings se establecen a partir de indicadores que de una manera sesgada y agregada ofrecen un valor final para cada ciudad que participa y sostiene económicamente 
las operaciones estadísticas. Luego cada informe individualizado se vende a un precio desorbitado para todas aquellas personas interesadas en justificar su cota de importancia en el listado (en este caso a 250 \$ cada uno).

Según las estadísticas oficiales del US Census Bureau, en 1980 Baltimore tenía 786.775 habitantes mientras que en 2009 los efectivos se redujeron un 18,7\% hasta caer a los 651.154 habitantes. En ese último año las viviendas vacías llegaban al 20\% (para una media en todo el país del $11,8 \%$ ) y el $20 \%$ de la población se encontraba por debajo del nivel de pobreza. En Pittsburgh, la ciudad norteamericana más habitable para The Economist, la población se redujo en ese período de tiempo un $26,1 \%$, de los 423.938 habitantes a los 313.118 , con un $16 \%$ de viviendas vacías y el $22 \%$ de la población por debajo del nivel de pobreza en 2009. Finalmente, Cleveland, la joya de Ohio, pasó de los 573.822 habitantes de 1980 a los 439.013 de 2009 (una reducción del 23,5\%), con un nivel de renta per cápita ese último año de 16.581 frente a los 27.041 \$ de media nacional, con el 20\% de las viviendas vacías y el $30 \%$ de la población total por debajo del nivel de pobreza.

Las preguntas podrían ser, ¿la crisis mundial motivó estos auténticos fracasos en la estrategia de crecimiento urbano?, o bien, ¿son estos ejemplos en realidad una parte más de la propia gestación de la crisis inmobiliaria mundial?, ¿cuantas nuevas viviendas se crearon en los procesos de espectacularización de las principales ciudades mundiales? y ¿a qué precios?, o incluso, ¿porqué una revista que defiende las políticas neoliberales como The Economist, mantiene en los primeros puestos del ranking de habitabilidad a ciudades que atraviesan una profunda crisis socioeconómica?.

La revalorización de la capacidad de los territorios para competir se produjo en el marco de la globalización, cuando las nuevas políticas urbanas en el marco de las gobernanzas locales «desregularon» el mercado y magnificaron la pugna establecida por hacerse más atractivas para captar más inversiones, siguiendo la lógica neoliberal de que un incremento en la competitividad se traduciría inmediatamente en mayores tasas de crecimiento (Lois, 2010).

El resultado es la creación de una ciudad fragmentada y polarizada socialmente. Los analistas urbanos emplean diferente terminología para referirse a los efectos de la globalización, se habla de ciudad dual, cuarteada, fragmentada, archipiélica, multifragmentada, polarizada, segregada, dividida, en peligro, informal e informacional. En definitiva, de la fractura social impuesta a través de las políticas locales y la transformación y gentrificación de amplios sectores urbanos, siguiendo instrumentos de planificación estratégica y modelos de gobernanza.

En la tesis del arquitecto Juan Carlos Etulain sobre el proyecto de renovación urbana de Puerto Madero en Buenos Aires (Etulain, 2008), la hipótesis inicial partía de como un proyecto de este tipo, bien dirigido desde el gobierno democrático local, podía incorporar mecanismos e instrumentos que posibilitaran al sector público actuar no sólo como inductor y controlador del proceso, sino como actor director sobre el que podían revertir parte de las plusvalías, que después se convertirían en «inversión ciudad» dirigida a otros sectores con déficit urbano y ambiental. Dieciocho años después, el doctor arquitecto concluía que las escasas instancias de participación ciudadana, la falta de realización de viviendas y equipamientos públicos, junto a la descapitalización sufrida por la corporación local y las operaciones meramente especulativas del sector privado tipificaban al proyecto como un modelo de privatización de la gestión urbanística, que construyó finalmente un enclave social de elite, un ghetto, una isla en el medio de un mar degradado y en peores condiciones que antes de 
que se iniciara este proyecto, una nueva ciudad sobre 170 hectáreas en un área urbana de interés para el mercado inmobiliario local e internacional (Etulain, 2008).

Como afirman varios estudios realizados sobre otras ciudades (Vicario y Martínez, 2003; Moulart et al, 2003; González, 2007; García, 2009) las grandes ciudades están siendo gobernadas mediante políticas urbanas de corte neoliberal con las que las corporaciones locales se convierten en un actor principal en la sustentación del mercado, generando desigualdades y una sociedad fuertemente polarizada.

En España han surgido diversas voces críticas especialmente contra los dos ejemplos más publicitados de renovación urbana posmoderna: el Modelo Guggenheim de Bilbao (Rodríguez, Moulaerte y Swyngedouw, 2001, Vicario y Martínez, 2003, González, 2004; Plaza et al, 2010; Mas, 2011) o el denominado modelo Barcelona (Capel, 2005; Casellas, 2006; Delgado, 2007; Borja, 2009). En estas investigaciones se cuestionan abiertamente los logros conseguidos en los últimos años por los procesos de renovación urbana y de gobernanza de ambas ciudades, denunciando como son los intereses del capital y especialmente del negocio inmobiliario los principales beneficiarios de las ingentes inversiones públicas canalizadas por los diferentes niveles administrativos para la «reinvención» de Barcelona y Bilbao, mientras que la mayoría de los habitantes se convierten en víctimas de procesos de segregación espacial por el alza continua de los precios.

A comienzos de la segunda década del siglo XXI, la crisis inmobiliaria y financiera mundial ha repercutido en el desarrollo de cientos de proyectos de transformación urbana de grandes magnitudes en ciudades de todo el mundo. Las grandes obras se han ralentizado y un ejemplo paradigmático es Dubai, el escaparate de las nuevas ciudades de la apariencia y la espectacularización urbana, enclavada en el desierto y el auténtico paraíso del lujo y la exclusividad dentro de un sistema social semifeudal.

En resumen, el resultado de los grandes proyectos urbanos y las experiencias de «gobernanza» urbana fueron positivos para el capital, las inmobiliarias y las elites socioeconómicas, que crearon una nueva ciudad en exclusiva y a su gusto. Visto de esta forma, el proceso es realmente un calco de lo que ocurrió en Europa y América del Norte a finales del siglo XIX y comienzos del XX con la creación de la ciudad burguesa, a veces «interpretando» y modificando a su antojo proyectos democráticos y humanistas como el ensanche de Barcelona o la ciudad jardín de Howard. Nuevas ideas de la forma urbana que nacían para mejorar la calidad de vida de sus habitantes y que al final se restringieron en exclusiva a las clases privilegiadas. Si bien la magnitud de las cifras económicas necesarias para las grandes operaciones contemporáneas supera exponencialmente a los «empréstitos», «emprendimientos» y «proyectos de ensanche»o «Haussmanización» de la ciudad novecentista.

\section{CRISIS Y RENOVACIÓN URBANA EN LAS CIUDADES MEDIAS ESPAÑOLAS}

En España, los ejemplos más evidentes de procesos de transformación urbana auspiciados por nuevos sistemas de gobernanza y planes estratégicos de competitividad se circunscriben a los ejemplos de Bilbao y el modelo de desarrollo impulsado por el Guggenheim, Barcelona y la renovación de la fachada marítima con las Olimpiadas y el Forum, Valencia y su Ciudad de las Artes y las Ciencias renovando completamente un barrio marginal, Zaragoza con el impulso renovador generado por la Exposición Universal de 2008 y Sevilla a 
partir de la renovación de Isla Mágica tras la Exposición Universal de 1992. Madrid, por su parte, ha desbordado sus procesos de especulación inmobiliaria en la periferia metropolitana, a la espera de contar con un gran evento como la organización de unos juegos olímpicos para actuar de forma integral sobre un gran sector de la ciudad central, pudiendo poner únicamente como ejemplo reciente el nuevo skyline que proporcionan las cuatro torres del CTBA, construidas sobre los campos de fútbol de la antigua ciudad deportiva del Real Madrid.

Ahora bien, ¿qué ocurre en el resto del sistema urbano? Para responder a este interrogante podemos referirnos a las ciudades medias, aunque para ello deberemos primeramente caracterizar este concepto.

Las ciudades medias son aquellos asentamientos urbanos que contienen un número de habitantes determinado y cumplen ciertas funciones de centralidad y reequilibrio territorial (Zoido et al., 2000). El umbral de población es variable, según la Ley 7/85 de Bases de Régimen Local estaría en España entre 20.000 y 100.000 habitantes, aunque otros autores incrementan este intervalo, situándolo entre 30.000 y 300.000 habitantes (López, 2010). También varían los límites demográficos en función de los países y así por ejemplo en Francia se sitúan entre 20.000 y 100.000 habitantes, en Alemania entre 50.000 y 100.000 o de forma genérica para la Unión Europea entre 20.000 y 200.000 habitantes (Zoido et al., 2000; López, 2010).

A este criterio cuantitativo se les unen otros dos cualitativos, en cuanto se supone que una ciudad media tiene un carácter de centralidad para el territorio que organiza, pudiendo por tanto hablar de área periurbana o incluso región urbana, y una posición de intermediación en el sistema de ciudades en el que se inscribe, entre las ciudades grandes, las ciudades pequeñas y el sistema complementario de asentamientos (López, 2010), por lo que a veces también se les denomina ciudades intermedias, destacando su importante papel como puntos de equilibrio del sistema de poblamiento para frenar la metropolización y el total abandono de los espacios periféricos.

Tradicionalmente se asocia a las ciudades medias una imagen de calidad de vida y desarrollo a escala humana y gracias a su posición intermedia han sido objeto de atención preferente de las políticas públicas encaminadas a favorecer procesos de crecimiento descentralizados (Zoido et al., 2000), como sucedió en 1999 con la aprobación de la denominada Estrategia Europea de Desarrollo Territorial, basada en tres pilares fundamentales, el desarrollo de un sistema urbano policéntrico y equilibrado en el marco de unas nuevas relaciones entre el espacio urbano y el rural, la garantía de acceso paritario a las infraestructuras y al conocimiento para todos los ciudadanos independientemente de su lugar de residencia, y la protección de la naturaleza y el patrimonio cultural.

En un reciente estudio sobre las ciudades medias españolas (Escudero y Somoza, 2009) se evaluaba su nivel de desarrollo en el período 1996-2006, para poder establecer un balance de lo que había supuesto para estas ciudades la Estrategia Territorial Europea. Para determinar esta evolución se empleó un índice compuesto denominado ODI por sus siglas en inglés (Overall Development Index, índice agregado de desarrollo), en el que se reunían datos demográficos, laborales, comerciales, económicos e inmobiliarios, concluyendo que sólo aquellas ciudades medias que se situaban en la periferia de una gran urbe, especialmente en el área metropolitana de Madrid y Barcelona, o las que tenían una especialización funcional turística, experimentaron en ese período un crecimiento destacable. Por el contrario, las ciudades capi- 
tales de provincia del interior mostraban un claro estancamiento. De las 112 ciudades analizadas, las que obtenían un menor índice eran en su mayoría capitales del interior y ciudades industriales como Avilés, Getxo, Ponferrada, Alcoi o Puertollano. Precisamente aquellos lugares que en los últimos años aprobaron proyectos estratégicos para renovar la imagen urbana y atraer inversiones, a través de la «gobernanza», la protección del patrimonio construido, la promoción del conocimiento y la asunción de compromisos de desarrollo sostenible. Unas medidas que indudablemente no generaron el éxito aguardado en los objetivos señalados, pero incrementaron, también sin lugar a dudas, la calidad de vida de sus habitantes (Escudero y Somoza, 2010). No obstante, no todas las ciudades medias han optado por desarrollar las mismas estrategias de renovación urbana. Si escogemos tres casos concretos fácilmente comparables y próximos entre sí, como Avilés, Ponferrada y Pontevedra, podemos establecer una tipología con los tres modelos más comunes desarrollados en los últimos años.

Avilés es una ciudad industrial que forma junto a Oviedo y Gijón y otros asentamientos menores el sistema urbano asturiano. Según la revisión de Padrón contaban en 2010 con 84.202 habitantes, lo que suponía un pequeño descenso con respecto a la población de 1991 de $-1,34 \%$. Vinculada como ciudad industrial a la siderúrgica ENSIDESA (hoy ArcelorMittal) ha sufrido una intensa crisis desde los años 1980, que se concreta espacialmente en el entorno industrial de su ría.

Para renovar su imagen urbana y siguiendo casi miméticamente el modelo de BilbaoGuggenheim-Ría del Nervión, el gobierno autonómico ofreció a la corporación municipal (PSOE-IU) en 2006 la posibilidad de desarrollar el proyecto donado por el arquitecto brasileño Óscar Niemeyer para albergar el museo de los Premios Príncipe de Asturias, distinción de prestigio internacional que el arquitecto había recibido en 1989. Los diseños de los edificios fueron un regalo expreso de Niemeyer al Principado de Asturias con motivo del 25 aniversario de estos Premios y cuando se empezó a gestar el proyecto surgieron los primeros problemas, tanto en cuanto a la funcionalidad final de los edificios, pues Oviedo se opuso frontalmente a «descentralizar» los Premios Príncipe de Asturias, como en cuanto a la participación del gobierno regional, pues Gijón reivindicaba un trato similar si se iba a empeñar en la renovación de Avilés un presupuesto importante procedente de las arcas autonómicas, además de que tampoco todos los habitantes de esta última ciudad tuvieran muy claro la necesidad y aprovechamiento resultante del proyecto.

En marzo de 2011 fue solemnemente inaugurado el Centro Cultural Óscar Niemeyer, ya sin ninguna referencia a los premios Príncipe de Asturias, como el primer paso de un ambicioso plan estratégico de renovación de la ría de Avilés, primero denominado «Nueva Centralidad» y posteriormente «Avilés, Isla de la Innovación» que se extiende por un gran espacio de 57'5 hectáreas y que incluye diversos proyectos residenciales que tardarán en materializarse, pero que parecen adivinarse en la fotografía.

Hoy en día podemos incluir este ejemplo de renovación dentro de los planes estratégicos de espectacularización urbana, en cuanto proceso que escoge una zona marginal susceptible de soportar un proceso intenso de vaciado para ubicar sobre el nuevo espacio «haussmanizado» una obra de arte arquitectónica «per se», un nuevo referente visual en el que prevalece la forma a la función, en el que importa más el continente que el contenido, pues no pasa nada si se modifica la idea inicial y se acaba definiendo un espacio cultural polifuncional en lo que antes iba a ser el Museo Nacional de los Premios Príncipe de Asturias. De hecho, 
Figura 1

CENTRO CULTURAL INTERNACIONAL EN LA RÍA DE AVILÉS

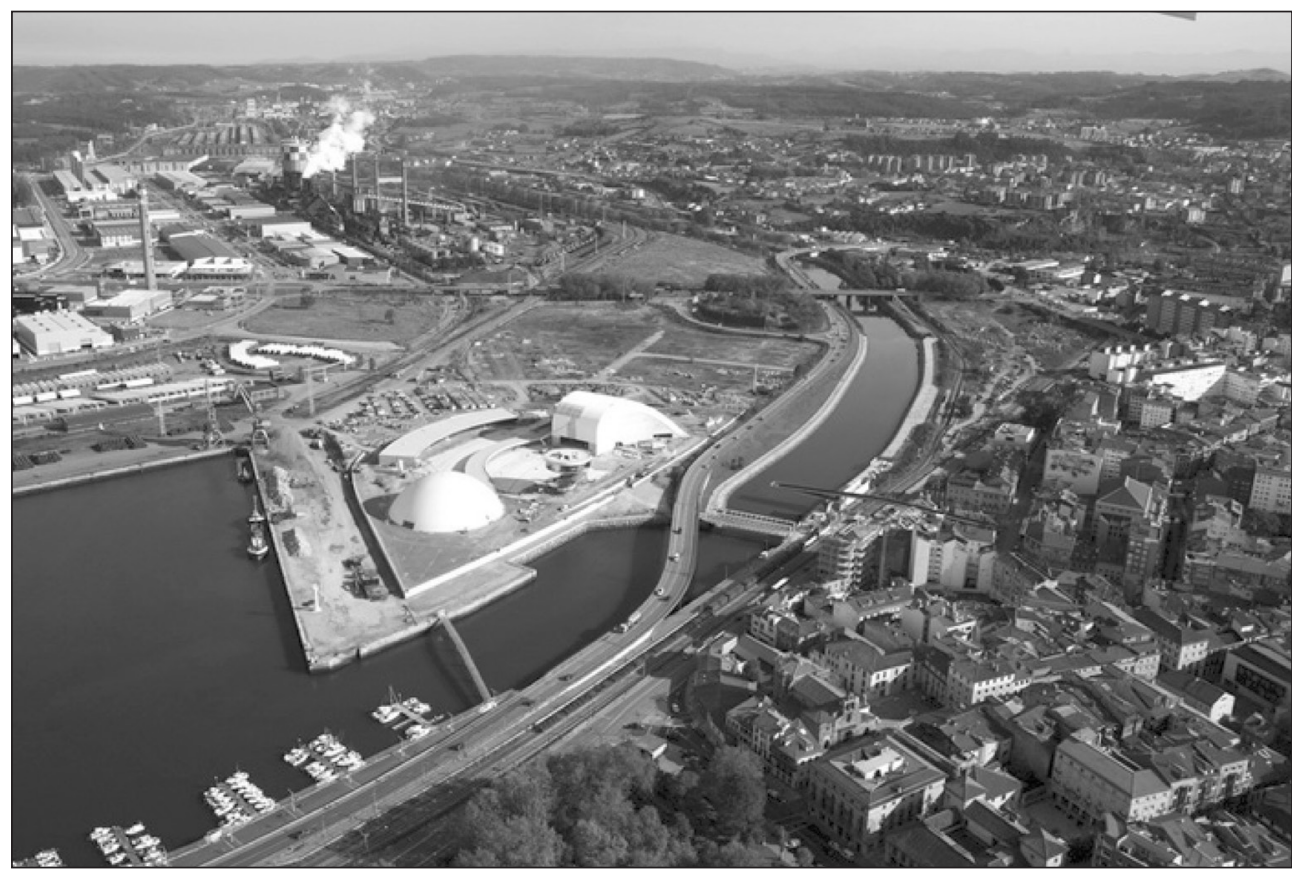

Fuente: www.niemeyercenter.org

y tras las elecciones autonómicas, la Fundación Oscar Niemeyer anunció que cerraría sus puertas el 15 de diciembre de 2011 por falta de financiación del nuevo gobierno autonómico presidido por Francisco Álvarez Cascos (FAC). La reapertura posterior significó una nueva reorientación y otro cambio de denominación, ahora Centro Cultural Internacional Avilés.

Para la población local, el Centro Cultural es motivo de orgullo y supondrá una mejora evidente en la oferta cultural de la ciudad, aunque suponga un gran esfuerzo para sus gestores mantener un calendario de actividades, exposiciones y espectáculos a lo largo de todo el año, contando con un público potencial tan reducido. Sólo el transcurrir de los próximos años podrá valorar de una manera objetiva si la inversión realizada ha servido para cumplir los ambiciosos objetivos de este proyecto de renovación urbana. Lo cierto es que las elecciones municipales de 2011 mantuvieron en el poder a la alcaldesa del PSOE, pero perdiendo un $15,5 \%$ de los votos obtenidos en los anteriores comicios.

Ponferrada, la capital de la comarca de El Bierzo, en el extremo noroccidental de Castilla y León, contaba en 2010 con 68.767 habitantes, lo que suponía respecto a 1991 un incremento del $14,7 \%$. La principal función industrial de Ponferrada estaba hasta hace poco supeditada a la Empresa Minero Siderúrgica de Ponferrada, MSP, que contaba al oeste del sector urbano más moderno (La Puebla), con unas extensas propiedades que incluían la denominada «montaña de carbón», un icono visual difícil de olvidar para todas aquellas personas que mientras estuvo activa la MSP visitaron la ciudad. 
Hoy en día, parte de las instalaciones de la MSP albergan el Museo Nacional de la Energía, pero la mayor parte del anterior espacio industrial se ha convertido en viviendas, entre las que el plan parcial de La Rosaleda merece una explicación más detallada.

El comienzo de este plan estratégico de renovación urbana se sitúa en el año 2000, y contó con la anuencia de la corporación municipal (PP) y el gobierno autonómico, que aprobó la reforma del plan general en 2001, aunque las continuas irregularidades, denuncias y sentencias de los diferentes tribunales, incluida una de 2007 del TSJ de Castilla y León contra esa misma modificación, ralentizaron su puesta en desarrollo.

La montaña de carbón sirvió, entre otras cosas, para nivelar el propio terreno de la actuación de la Rosaleda, extendiendo toneladas de estériles sobre una superficie de 65 hectáreas. El plan aprobado por la corporación municipal incluía la construcción, sólo en este sector, de casi 5.000 viviendas, lo que suponía un 16,4\% del total de viviendas registradas en el municipio en 2001, pero además, borrada de la memoria colectiva la imagen de la montaña artificial, los gobernantes pensaron que debería ser reemplazada por otra estructura similar, edificándose la Torre Rosaleda, el edificio de viviendas más alto de Castilla y León, con 107 metros de altura y 28 plantas.

Una de las principales empresas implicadas, tanto en la construcción de la torre, como en la del centro comercial La Rosaleda y otros edificios de viviendas en el sector fue BEGAR, propiedad de José Luis Ulibarri, la gran empresa constructora leonesa junto a Martínez Núñez, impulsora en la capital provincial de otra gran actuación semejante en el polígono de La Lastra con 4.700 viviendas, aunque sin rascacielos. Ambas empresas también comparten una implicación directa en el denominado caso Gürtel de corrupción política y atraviesan importantes dificultades financieras.

Figura 2

EL NUEVO BARRIO RESIDENCIAL DE LA ROSALEDA EN PONFERRADA CON SU RASCACIELOS

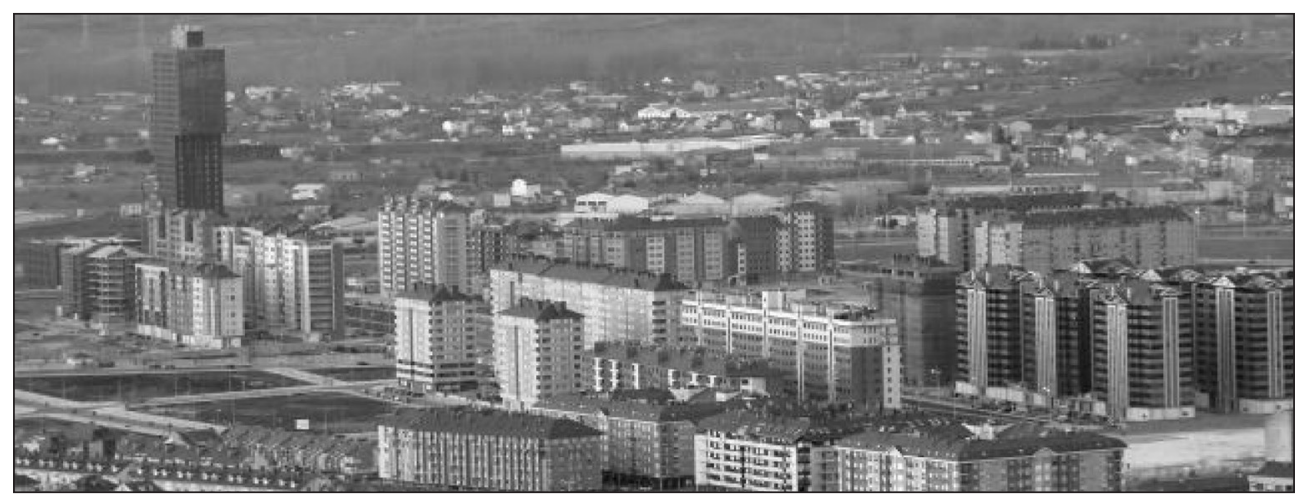

Fuente: www.publico.es

Puede que en el futuro cambie la situación, pero hoy en día La Rosaleda es un enorme espacio residencial con multitud de viviendas y locales vacíos, como ocurre en la población toledana de Seseña, eso sí, la torre de La Rosaleda, el monumento a la especulación inmobiliaria de una ciudad de 68.000 habitantes se puede observar a varios kilómetros de distancia. 
Al igual que en el caso anterior, la reválida que suponen las elecciones municipales mantuvieron en el poder al alcalde ponferradino, si bien perdió el $22 \%$ de los apoyos obtenidos en 2007 y la mayoría absoluta que ostentaba desde entonces.

Pontevedra es una ciudad capital de provincia, que vivió durante el siglo XX a la sombra del desarrollo de la vecina ciudad industrial de Vigo. En torno a una ría con dificultades por la instalación en 1963 de una industria de celulosa en sus proximidades, los alcaldes de los últimos años han accedido al sillón presidencial con la consigna de expulsar a ENCE de la ría, aunque pasado el tiempo la fábrica sigue allí, con mejoras introducidas en su ciclo productivo pero sin que se pueda erradicar de este lugar una industria tan contaminante.

Pontevedra contaba en 2010 con 81.981 habitantes, lo que suponía el mismo porcentaje moderado de incremento respecto a la población de 1991 que registraba Ponferrada $(14,7 \%)$. Durante toda la segunda mitad del siglo XX el centro histórico de la ciudad fue perdiendo población y convirtiéndose en un espacio marginal, como ha ocurrido en gran número de ciudades en España, al tiempo que el sector urbano moderno y central se saturaba de población y actividades económicas con los consecuentes problemas de tráfico que este desplazamiento gravitacional significaba.

La corporación municipal que llegó al poder en 2003 (BNG-PSOE), decidió realizar un plan estratégico de renovación de la ciudad atendiendo a dos líneas fundamentales, recuperar el centro histórico como espacio económico y residencial y reducir el tráfico en el centro urbano para mejorar la calidad de vida de sus habitantes. El primer paso supuso una considerable inversión de esfuerzo y recursos, pero consiguió recuperar el orgullo de la población por el sector que acumula la memoria histórica de la ciudad, su identidad, y reactivar económicamente y demográficamente este barrio, ahora reconocido siguiendo estrategias de marketing urbano como «la ciudad de piedra». Para el segundo paso fue fundamental la comprensión y colaboración de la ciudadanía, pues reducir el tráfico motorizado supone soportar inicialmente ciertas molestias hasta que se modifican los hábitos de desplazamiento, primando el transporte público y la movilidad peatonal. El resultado de este segundo proceso fue reconocido en 2010 con el premio nacional de seguridad vial.

En el ejemplo de Pontevedra, a diferencia de los otros dos, el espacio a recuperar-renovar fue la propia ciudad. El espacio habitado que se había degradado en las últimas décadas por la falta de funciones o su excesiva concentración. Pontevedra es hoy en día una ciudad media que ha ganado considerablemente en habitabilidad como reconocen abiertamente sus ciudadanos y ciudadanas, y todo ello sin contar con un espacio espectacular de un arquitecto o arquitecta de fama internacional ni un nuevo skyline provocado por la especulación inmobiliaria.

Al igual que en Avilés o Ponferrada, tras las elecciones de 2011 continúa el mismo alcalde (BNG), pero en este caso los apoyos han subido en estos cuatro años un $38 \%$.

Tres modelos por tanto de renovación urbana, procesos de espectacularización que necesitan de grandes inversiones de todas las administraciones públicas, especulación inmobiliaria en la que participan activamente las empresas constructoras con el apoyo de las corporaciones políticas o medidas de menor impacto visual y que necesitan más tiempo para tornar más habitables nuestras ciudades pensando fundamentalmente en la ciudadanía. 
Figuras 3 y 4

RECUPERACIÓN EN EL CENTRO HISTÓRICO DE PONTEVEDRA Y MEDIDAS DE PEATONALIZACIÓN
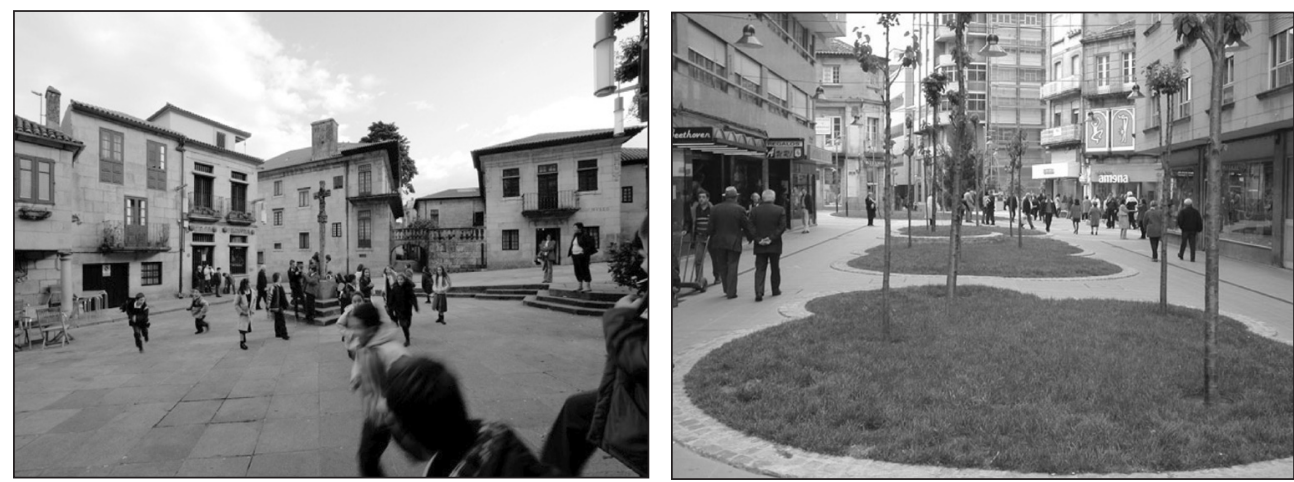

Fuente: www.concellopontevedra.es

\section{CONCLUSIONES}

Como comprobamos en este artículo, la gobernanza no es un término inocente e inocuo. Se trata de un concepto ideologizado, que parte de las escuelas de pensamiento neoliberal estadounidenses y que con el paso del tiempo se ha extendido por todos los ámbitos institucionales de gobierno, desde las organizaciones de cooperación internacional a las corporaciones locales, pasando por los organismos supranacionales y los gobiernos estatales y regionales. La gobernanza pretende normalizar la creación de estructuras relacionales público-privadas para dirigir las políticas, proyectos y gestiones de las instituciones democráticas.

En el caso de la gobernanza local, el escenario viene marcado por el cambio en las políticas urbanas que ahora adoptan un estilo proactivo, dinámico y empresarial, buscando oportunidades donde el sector público y el privado puedan concertar acciones conjuntas que incrementen la competitividad urbana.

La expresión física de la nueva planificación estratégica y la gobernanza urbana son los grandes proyectos de transformación urbana, que se inician en determinadas ciudades de los Estados Unidos y se convierten desde los años 1990 en una constante en las principales urbes del mundo.

En estos proyectos urbanísticos, los gobiernos lideran de forma proactiva una nueva política económica basada en grandes intervenciones estelares, promoviendo las inversiones privadas por medio de instrumentos de apoyo directo e indirecto, incentivos fiscales y costosas campañas de marketing urbano.

No obstante, casi veinte años después de iniciados muchos de estos grandes procesos de transformación urbana, podemos comprobar como los casos de éxito son muy escasos, e incluso en los más publicitados, la realidad esconde una situación mucho más compleja, a pesar de figurar en puestos destacados en los rankings mundiales de habitabilidad.

La «gobernanza» urbana entendida bajo este prisma y su expresión urbanística, los grandes proyectos de renovación de las ciudades, siguen siempre un mismo patrón y acaban, 
igualmente, provocando los mismos resultados, la segregación socioespacial de la ciudad, la fractura entre el espacio urbano globalizado del diseño y la opulencia y el resto del entramado físico y social.

Las ciudades intermedias presentan en España una evolución reciente complicada, a juzgar por los datos socioeconómicos y en comparación con el crecimiento sostenido de las grandes áreas metropolitanas y la costa mediterránea, fuertemente turistificada. Ante esta situación es fácil caer en los discursos proactivos de transformación económica y dinamismo futuro, aunque en una coyuntura como la actual será difícil que las grandes compañías del sector inmobiliario seduzcan a las corporaciones locales con cantos de sirena.

Patrick Le Gales afirmaba en una conferencia (Le Gales, 2007) que existían en Europa dos dinámicas diferentes de gobernanza. Por un lado un modelo de asociación e intercambio entre muchas organizaciones que llevaba a un incremento de la burocratización, y por otro, muchos intentos de restablecer elementos de democracia y concertación con los ciudadanos, lo que para él era la verdadera dimensión política y democrática de la participación ciudadana. Este sería para algunos el denominado principio de subsidariedad social u horizontal, según el cual debe favorecerse la iniciativa y la participación de los ciudadanos y ciudadanas, individual y colectivamente, para la realización de actividades de interés general. Gobernar incluye entonces formas de empoderamiento social (Rojo, 2009; Romero y Farinós, 2011).

Así muchas ciudades medias con una agradable calidad de vida, que apostaron por la sostenibilidad y la integración, por el bienestar social y la implicación ciudadana, acabaron por configurar realidades muy competitivas frente a propuestas grandilocuentes, espectaculares y escenográficas (Lois, 2010). Como explica Rubén Lois, esta idea formaría parte de un nuevo paradigma, antitético al neoliberalismo imperante, en el que el desarrollo humano y la calidad de vida de la personas constituyen los principales objetivos a conseguir por la ordenación territorial, substituyendo indicadores de competitividad urbana y potencialidad productiva por valores relacionados con la satisfacción y el ejercicio de derechos ciudadanos (Lois, 2010). Se trata entonces de planificar la ciudad en función de los intereses de su población, no de los que defienden las grandes empresas multinacionales o las agencias inmobiliarias, de fijarse en las capacidades productivas endógenas y no tratar de atraer nuevas funcionalidades, de satisfacer las demandas de las vecinas y vecinos no las de los posibles visitantes, de favorecer, en suma, la felicidad de la ciudadanía mejorando su bienestar y no generar ilusiones infundadas basadas en imágenes y slogans publicitarios (Somoza, 2007).

Si creemos en esta forma de gobernar y proyectar urbanísticamente nuestras ciudades debemos desarrollar una incesante labor de pedagogía social. Es difícil abstraerse de un discurso de grandes proyectos grandilocuentes cuando la oportunidad llega a una ciudad, sea en forma de nueva estación para el AVE, plataforma ciudadana para el nombramiento como capital europea de la cultura, nuevo campus universitario de excelencia o el gran paseo en la fachada marítima o fluvial. Lo importante en estos casos es no dejarse llevar por las cifras económicas ni por la necesidad de financiar las operaciones con promociones inmobiliarias, y realizar una planificación a medio plazo con criterios de excelencia, valorando los resultados a través de indicadores que analicen la reducción de la pobreza, la redistribución de la riqueza, o el acceso a los servicios básicos y la sostenibilidad de las actuaciones realizadas (Lois, 2010). 
En los próximos años les tocará a muchos gobiernos locales de las ciudades medias españolas decidir qué tipo de transformación urbana acometen. Una repetición a escala de las grandes operaciones ya analizadas, como ocurre en Avilés o Ponferrada, o un cambio lento, a un ritmo diferente, que vela por los intereses de la ciudadanía y no del sector privado, como ocurrió en Pontevedra.

La única posibilidad a comienzos del siglo XXI de reconducir el desarrollo de las ciudades medias españolas es cambiar el modelo, adoptar un nuevo paradigma en el que el desarrollo humano y la calidad de vida de las personas constituyan los principales objetivos a conseguir, planificando la ciudad en función de los intereses de la ciudadanía.

Sólo de esta forma podremos habitar unas ciudades en las que el sistema de gobierno urbano recoja fórmulas de empoderamiento social, en las que los parámetros económicos se fundamenten en las potencialidades endógenas, en las que el urbanismo tenga en cuenta a las mujeres, a las personas mayores, a la juventud y a la infancia, y en las que el ecosistema cultural permita el desarrollo del talento y la creatividad sin olvidar las raíces que conforman su personalidad como pueblo. Unas ciudades más saludables, comprometidas con el medio ambiente y la sostenibilidad.

\section{BIBLIOGRAFÍA}

ALBRECHTS, L. (1992): «New challenges for urban policy under a flexible regime of accumulation», Landscape and Urban Planning, 22: 189-203.

ANDRÉS LÓPEZ, G. (2008): «Geografía y ciudades medias en España: ¿a la búsqueda de una definición innecesaria?», Scripta Nova, 270, 15 de enero de 2011. Universidad de Barcelona. Disponible en http://www.ub.es/geocrit/sn//sn-270/sn-270-49.htm.

ASHWORTH, G. y VOOGD, H. (1990): Selling the City. Londres. Belhaven Press.

BEVIR, M. y RHODES, R.A.W. (2010): The State as Cultural Practice. Oxford. Oxford University Press.

BLANCO, I. y GOMÁ, R. (2006): «Del gobierno a la gobernanza: oportunidades y retos de un nuevo paradigma». Politika. Revista de Ciencias Sociales, $\mathrm{n}^{\circ}$ 2/Diciembre, 11-27.

BORJA, J. (2009): Luces y sombras del urbanismo de Barcelona. Barcelona. UOC.

BORJA, J. y CASTELLS, M. (1997): Local y global. La gestión de las ciudades en la era global. Madrid. Taurus.

CAPEL SÁEZ, H. (2005): El Modelo Barcelona: un examen crítico. Barcelona. Ed. del Serbal.

CASELLAS, A. (2006): «Las limitaciones del «modelo Barcelona». Una lectura desde Urban Regime Análisis», en Documents d'Anàlisi Geogràfica, nº 48, pp. 61-81.

CENTELLES I PORTELLA, J. (2006): El buen gobierno de la ciudad. Barcelona. IIG.

COMISIÓN DE LAS COMUNIDADES EUROPEAS (2001): La Gobernanza Europea. Un libro blanco. Bruselas. Publicaciones de la Comisión de las Comunidades Europeas.

CONEJERO PAZ, E. (2005): «Globalización, gobernanza local y democracia participativa». Cuadernos Constitucionales de la Cátedra Fadrique Furió Ceriol, $\mathrm{n}^{\circ}$ 52/53, 13-31.

DAVIES, W.K.D. y TOWNSHEND, I. J. (eds.) (2002): Monitoring Cities: International Perspectives. Lethbridge. Graphcom Printers. 
DELGADO, M. (2007): La ciudad mentirosa: fraude y miseria del «modelo Barcelona». Barcelona. Los Libros de la Catarata.

ESCUDERO GÓMEZ, L. A. y SOMOZA MEDINA, J. (2010): «Medium-sized Cities: Polycentric Strategies vs the Dynamics of Metropolitan Area Growth» en The Open Urban Studies Journal, $\mathrm{n}^{\mathrm{o}}$ 2/13, 15 de enero de 2011. Oak Park, Bentham Open. Disponible en http://www.bentham.org/open/tousj/articles/V003/1TOUSJ-hot\%20topic/2TOUSJ.pdf

ETULAIN, J. C. (2008): «¿Gestión promocional o privatización de la gestión urbanística» en Bitacora $\mathrm{n}^{\circ}$ 12, 15 de enero de 2011. REDALYC. Disponible en http://redalyc.uaemex. $\mathrm{mx} /$ src/inicio/ArtPdfRed.jsp?iCve=74811916011

FARINÓS DASÍ, J. (2006): «Gobernanza territorial de ámbito metropolitano» en Los procesos Metropolitanos: materiales para una aproximación inicial (Feria Toribio, J. M., coord). Sevilla, Junta de Andalucía: 155-166.

FARINÓS DASÍ, J. (2008): «Gobernanza territorial para el desarrollo sostenible: estado de la cuestión y agenda». Boletín de la A.G.E., $\mathrm{n}^{\circ} 46,11-32$.

GARCÍA, G. (2009): «La gobernanza: el «buen gobierno» neoliberal». Sistema, no 212, 15-29.

GONZÁLEZ, S. (2007): «Trepando por la jerarquía urbana: nuevas formas de gobernanza neoliberal en Europa» en Ciudades en la sociedad de la información (Vivas, P.; Ribera, R.; González, F., coords), 15 de enero de 2011. UOC papers. Disponible en http://www. uoc.edu/uocpapers/5/dt/esp/gonzalez_sara.pdf.

GONZÁLEZ CEBALLOS, S. (2004): «The role of the Guggenheim Museum in the development of urban entrepreneurial practices in Bilbao», en International Journal of Iberian Studies $n^{\circ} 16(3)$, pp. 177-186.

HALL, P. (1988): Cities of Tomorrow. Oxford. Blackwell.

HALL, P. y HUBBARD, P. (Eds.) (1998): The Entrepreneurial City. Geographies of Politics, Regime and Representation. Chichester. Wiley.

HARVEY, D. (1989): «From managerialism to entrepreneurialism: the transformation of urban governance in late capitalism». Geografiska Annaler, $\mathrm{n}^{\circ}$ 71b, 3-17.

HAUS, M.; HEINELT, H. y STEWART, M. (Eds.) (2005): Urban governance and democracy. Londres. Routledge.

JESSOP, B. (1997): «The entrepreneurial city: re-imaging localities, redesigning economic governance, or restructuring capital?» en Transforming Cities (Jewson, N., MacGregor, S., Eds.). Londres. Routledge.

JOUVE, B. (2005): Cuestiones sobre gobernanza urbana. Barcelona. Fund. Cales Pi Sunyer. KOOIMAN, J. (ed.) (1993): Modern governance. New government-society interactions. Londres. Sage.

KOOIMAN, J. (2005): «Gobernar en gobernanza» en La gobernanza hoy: 10 textos de referencia (Cerrillo i Martínez, A., Ed.). Madrid. INAP.

LE GALES, P. (2007): «Gobernanza y cohesión social» en Congreso Regiones Capitales (Hall, P., dir.). Madrid. Comunidad de Madrid, 327-347.

LOIS GONZÁLEZ. R. C. (ed.) (2006): Urban Changes in Different Scales: Systems and Structures. Santiago de Compostela. Universidade de Santiago de Compostela e IGU.

LOIS GONZÁLEZ, R. C. (2010): «Ordenación territorial y competitividad interterritorial: una relación compleja» en Territorio. Ordenar para competir (Rodríguez González, R., Dir.). A Coruña. Netbiblo, 29-51. 
LÓPEZ TRIGAL, L. (2010): Diccionario de términos sobre la ciudad y lo urbano. Madrid. Biblioteca Nueva.

MARTÍNEZ BROUCHAR, M. F. (2010): «Gobernanza y legitimidad democrática». Reflexión Política, $\mathrm{n}^{\mathrm{O}}$ 23, 97-107.

MAS SERRA, E. (2011): «La revitalización del Área Metropolitana de Bilbao: la gestión de Bilbao Ría 2000, aspectos territoriales», en Boletín de la AGE, nº 55, pp. 35-57.

MAYNTZ, R. (2005): «Nuevos desafíos de la teoría de la gobernanza» en La gobernanza hoy: 10 textos de referencia (Cerrillo i Martínez, A., ed.). Madrid. INAP.

MOULAERT, F; RODRÍGUEZ, A. y SWYNGEDOUW, E. (eds.) (2003): The Globalized city. Economic Restructuring and Social Polarization in European Cities. Oxford. Oxford University Press.

MURAYAMA, Y. Y DU, G. (Eds.) (2005): Cities in Global Perspective: Diversity and Transition. Tokio. Rikkyo University e IGU.

PACIONE, M. (Ed.) (2004): Changing Cities. Glasgow. Strathclyde University Publishing e IGU.

PAK, M. (Ed.) (2004): Cities in Transition. Ljubljana. University of Ljubljana e IGU.

PASCUAL i ESTEVE, J. M. y GODÁS i PÉREZ, X. (Coords.) (2010): El buen gobierno 2.0. La gobernanza democrática territorial: ciudades y regiones por la cohesión social y una democracia de calidad. Valencia. Tirant lo Blanch.

PLAZA, B.; GONZÁLEZ-FLORES, A.; GÁLVEZ-GÁLVEZ, C. y MÁS, E. (2010): «Arte y Economía, un matrimonio de conveniencia: el museo Guggenheim en Bilbao» en Scripta Nova, XIV, n 335, 13 de abril de 201.1Universidad de Barcelona. Disponible en http://www.ub.edu/geocrit/sn/sn-335.htm

RHODES, R.A.W. (1996): «The new governance: Governing without government». Political Studies, $\mathrm{n}^{\circ}$ 44, 652-667.

RODRÍGUEZ, A.; MOULAERT, F. y SWYNGEDOUW, E. (2001): «Nuevas políticas urbanas para la revitalización de las ciudades en Europa». Ciudad y Territorio, $\mathrm{n}^{\circ}$ XXXIII (129), 409-424.

ROJO SALGADO, A. (2009): «Una aproximación al concepto de gobernanza» en Ordenación y gobernanza de las áreas urbanas gallegas (Rodríguez González, R., Dir.). A Coruña, Netbiblo, 3-27.

ROMERO, J. y FARINÓS, J. (2011): «redescubriendo la gobernanza más allá del buen gobierno. Democracia como base, desarrollo territorial como resultado». Boletín de la Asociación de Geógrafos Españoles, nº 56, 295-319.

ROSENAU, J. N. y CZEMPIEL, E. O. (Eds.) (1992): Governance without Government: Order and Change in World Politics. Nueva York. Cambridge University Press.

SOMOZA MEDINA, J. (2007): «Spanish virtual cities: Images, realities and city marketing» en Tourism and Urban Transformation (Du, G.,Ed.). Tokyo. Rykkyo University Press, 93-110.

VICARIO, L. y MARTÍNEZ MONJE, P. M. (2003): «Another Guggenheim effect?. The generation of a potentially gentrifiable neigbourhood in Bilbao». Urban Studies, $\mathrm{n}^{\circ} 40$, 2383-2400.

ZOIDO, F.; VEGA, S. de la; MORALES, G.; MAS, R. y LOIS, R. C. (2000): Diccionario de geografía urbana, urbanismo y ordenación del territorio. Barcelona, Ariel. 